\title{
On the scientific motivation for a wide field-of-view TeV gamma-ray observatory in the Southern Hemisphere
}

\section{Miguel Mostafá* for the HAWC collaboration ${ }^{\dagger}$}

E-mail: miguelepsu.edu

\begin{abstract}
Observations of high energy gamma rays are an essential probe of the cosmic-ray acceleration mechanisms because they are created by cosmic rays interacting near their origin. The characteristics of the gamma-ray flux variability and spectra constrain the acceleration mechanisms and the environment of the accelerator. Detection of the highest energy gamma rays and the shortest timescales of variability are key considerations when designing the next generation of gamma-ray experiments.
\end{abstract}

Instruments with a wide field of view and large duty cycle are capable of continuously surveying the very high energy gamma-ray sky, mapping the diffuse emission, detecting emission from very extended regions, and observing transient events such as gamma-ray bursts. They also have the potential for discovering electromagnetic counterparts to gravitational waves and astrophysical neutrinos. I will present the scientific motivation for a next-generation water-Cherenkov observatory located at very high altitude in South America.

Corresponding authors: S. Y. BenZvi ${ }^{1}$, M. Mostafá ${ }^{2}$, H. Schoorlemmer ${ }^{3}$, F. Schüssler ${ }^{4}$

${ }^{1}$ Department of Physics and Astronomy, University of Rochester, Rochester, NY, USA

${ }^{2}$ Department of Physics, Pennsylvania State University, University Park, PA, USA

${ }^{3}$ Max-Planck-Institut für Kernphysik, Heidelberg, Germany

${ }^{4}$ CEA Saclay, France

35th International Cosmic Ray Conference - ICRC2017

10-20 July, 2017

Bexco, Busan, Korea

${ }^{*}$ Speaker.

${ }^{\dagger}$ http://www.hawc-observatory.org/collaboration/icrc2017.php 


\section{High Altitude Water Cherenkov Observatory}

Observations of very high energy gamma-ray sources, especially above $10 \mathrm{TeV}$, require a large effective area and long integration times because the gamma-ray flux drops very rapidly as a function of energy. The point-source sensitivity of HAWC, shown in Fig. 1, is comparable to that of imaging air Cherenkov telescopes (IACTs) such as HESS and VERITAS after 50 hours of exposure. When comparing these two types of instruments, it is important to keep in mind that IACTs have a smaller field of view and can only operate on clear nights. In contrast, a HAWClike detector has a large field of view and can operate nearly $100 \%$ of the time. For example, HAWC observes every $\mathrm{TeV}$ gamma-ray source in the northern sky for 1500 hours every year [1]. These two types of detectors clearly complement each other. Having survey instruments in both hemispheres will allow us to combine both deep and focused observations on individual sources with a comprehensive survey of the whole sky.

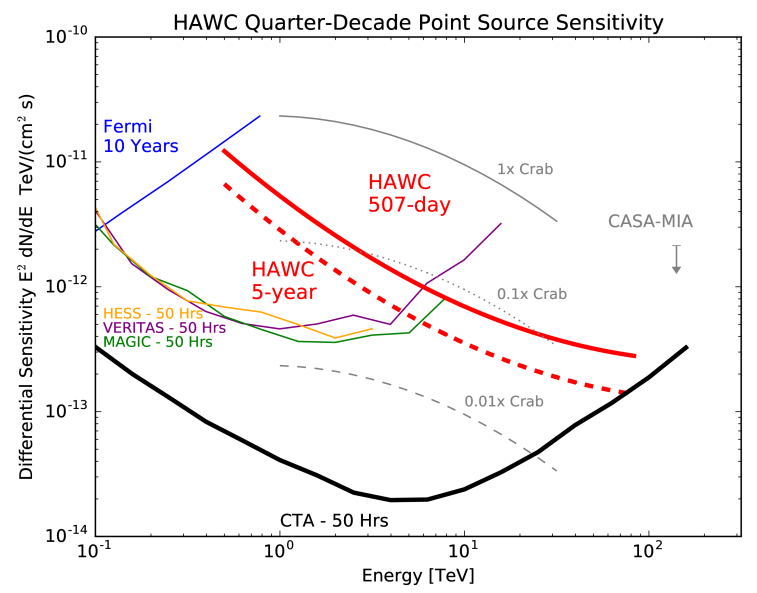

Figure 1: The quasi-differential sensitivity of HAWC to a point source at a declination of $+22^{\circ} \mathrm{N}$ as a function of photon energy, compared to existing IACTS, CTA, and the Large Area Telescope on the Fermi Gamma-Ray Space Telescope. Also shown is an inferred quarter-decade differential upper limit from the Crab at $141 \mathrm{TeV}$ from the CASA-MIA experiment. We show the flux, assuming a source with a differential energy spectrum $E^{-2.63}$, required to produce a $5 \sigma$ detection $50 \%$ of the time. The 507 -day observation of HAWC corresponds to $\sim 3000$ hours of a source at a declination of $22^{\circ}$ within HAWC's field-of-view. The one-year sensitivity of HAWC surpasses a 50-hour observation by current-generation IACTs at $\sim 10 \mathrm{TeV}$. From Fig. 16 in Ref. [1].

Air Cherenkov telescopes typically observe sources for less than $\sim 50$ hours, and survey observations last for about 10 hours. As shown in Fig. 1, the sensitivity of HAWC above roughly $10 \mathrm{TeV}$ is better than the sensitivity of 50 hours of observation of a single source with VERITAS or HESS. An IACT can only spend up to about 200 hours per year observing a single interesting source due to ambient light and weather constraints on telescope operations. Above $100 \mathrm{TeV}$, the sensitivity of HAWC is even comparable with the current expectation for CTA.

Most of the Galactic sources observed with HESS are not detected above $10 \mathrm{TeV}$. This is an important threshold because gamma-ray emission due to electron scattering of low-energy photons is expected to become inefficient at high energies. Sources with hard spectra above $10 \mathrm{TeV}$ are good candidates for the acceleration sites of protons and other charged particles. This kind of 

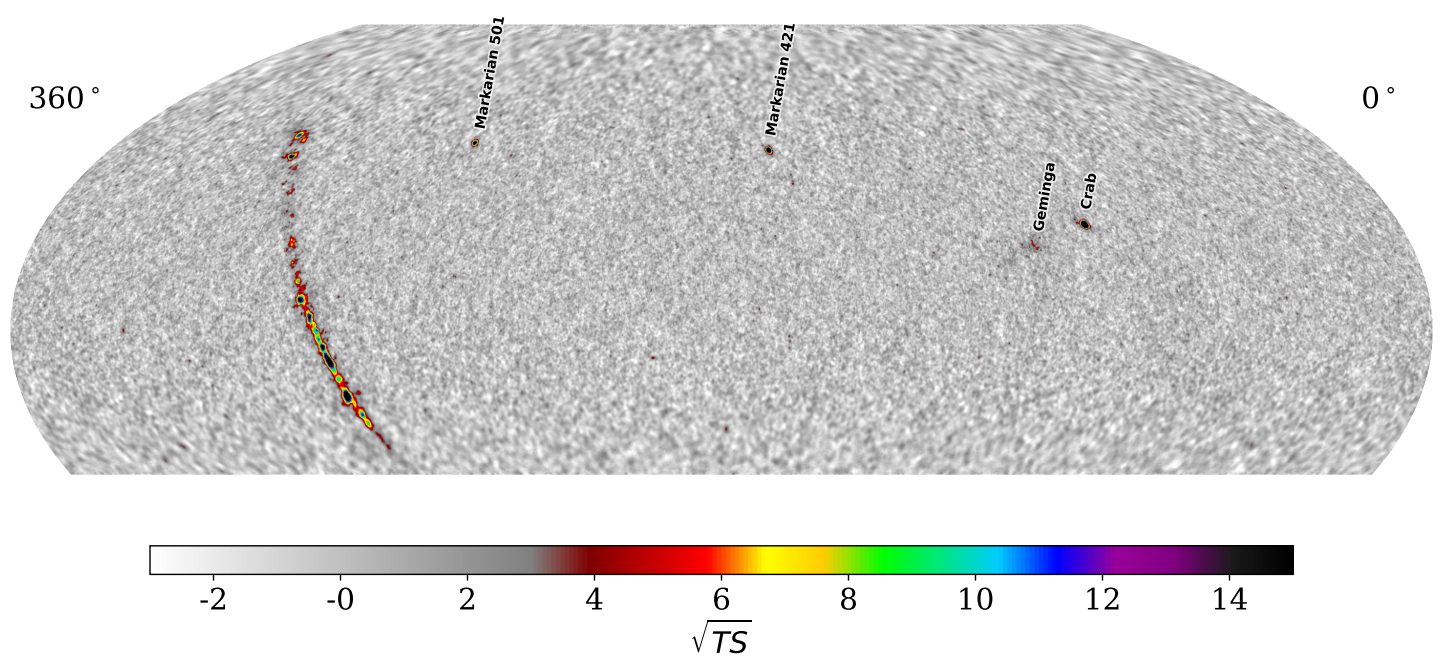

Figure 2: Equatorial full-sky map of significance, for a point-source hypothesis with a spectral index of -2.7. From Fig. 4 in Ref. [2].

discrimination between different particle acceleration models for sources with an exponential cutoff at a few tens of $\mathrm{TeV}$ is possible with HAWC-like detector. For example, HAWC will be able to measure 20 gamma rays above $100 \mathrm{TeV}$ from a source with a spectral index of -2.3 and $20 \%$ of the flux of the Crab Nebula after 5 years of data. Whether or not $100-\mathrm{TeV}$ gamma rays are observed will help us understand the acceleration processes and limitations at these sources.

\section{Studying Galactic Particle Accelerators}

How and where are cosmic rays accelerated? Cosmic rays up to at least $1 \mathrm{PeV}$, and perhaps as high as $1 \mathrm{EeV}$, are thought to be of Galactic origin. The observed spectral break at PeV energies may be due to the spectrum of cosmic-ray sources, the escape of the cosmic rays from the Galaxy, or a combination of the two effects. It has also been speculated that a single nearby source could be the cause of the knee. Interactions between protons with energies of about $1 \mathrm{PeV}$ and molecular clouds or other matter can produce $\sim 100 \mathrm{TeV}$ gamma rays. These very high energy gamma rays are observable with HAWC in the northern hemisphere from both point and extended sources, as well as from the sea of cosmic rays interacting with matter in the Galactic plane.

Supernova remnants (SNRs) have been postulated as the origin of Galactic cosmic rays largely because they have sufficient energy to provide the observed local cosmic ray energy density. SNRs also have sufficiently strong magnetic fields to trap particles long enough to accelerate them up to at least $100 \mathrm{TeV}$. Multi-TeV gamma rays have been observed from SNRs, but also from other Galactic sources, such as pulsar wind nebulae and compact binary systems. Which of these sources accelerate hadronic cosmic rays? What is the total power output of these Galactic accelerators? 
Observations of many different types of sources at the very highest energies are essential to answer these questions.

Gamma-ray observations at the highest possible energies are key to distinguishing gamma rays produced by electrons from those produced by hadrons. There are observational differences in the $\mathrm{TeV}$ gamma-ray spectrum from electron accelerators and proton accelerators accessible to a HAWC-like detector. Electrons lose their energy more quickly than protons due to synchrotron emission and are therefore more difficult to accelerate to the highest energies. Also, the cross section for inverse Compton scattering decreases at higher energies, resulting in a break in the gamma-ray spectrum at a few tens of $\mathrm{TeV}$. Gamma rays from hadronic cascades in the accelerating region, on the other hand, follow the power law spectrum of the particles initiating the cascades up to the highest energies.

Therefore, a primary goal of a HAWC-like detector is to perform careful measurements of the fluxes of $\mathrm{TeV}$ sources up to $100 \mathrm{TeV}$. Sources with very hard spectral indices that do not exhibit sharp cutoffs in the multi-TeV range are good candidates for hadronic acceleration. The proposed survey instrument will observe the accelerators in the southern sky. In particular, the center of the Galaxy (outside the field of view of HAWC) would transit directly overhead.

\subsection{Extended emission}

Many of the $\mathrm{TeV}$ gamma ray sources detected with HESS in the Galactic plane are spatially extended; i.e., larger than the point spread function of the telescopes of about $0.1^{\circ}$. The source region itself could be extended, or the observed extension could be due to the interactions of cosmic rays with material in the extended vicinity of their acceleration sites. For example, HESS measurements of the $\mathrm{TeV}$ emission from the W28 supernova remnant indicate that the molecular clouds surrounding the remnant also emit $\mathrm{TeV}$ gamma rays. This emission could be due to the interaction of cosmic rays with the material inside the clouds.

Although IACTs are quite sensitive instruments, it is difficult to observe the full extent of large sources with instruments with a narrow field of view. When the size of a source is much larger than the point spread function of an imaging air Cherenkov telescope, the sensitivity of the detector worsens because the background increases. Current observations of extended sources with HESS are clustered around the limit of their sensitivity, suggesting the presence of even more extended sources. In particular, nearby sources may have a larger angular extent than currently reported by IACTs. One such nearby source is Geminga, a supernova remnant that is located several hundred parsecs from Earth. The Milagro collaboration has reported evidence of $\mathrm{TeV}$ emission from a $2.9^{\circ}$ wide region around Geminga, which corresponds to a physical size of about $8 \mathrm{pc}$. The HAWC observations of the region around Geminga are shown in Fig. 3. With even higher sensitivity, it should be possible to estimate the energy spectrum as a function of angular distance to the center of the pulsar. The highest energy electrons should lose energy quickly as they propagate away from the source; while this is not true for protons. Therefore, if Geminga were an electron accelerator, we would see a clear change in the spectral index as we look farther from the source.

\subsection{Diffuse emission}

Observations of the $\mathrm{GeV}$ and $\mathrm{TeV}$ sky have shown that the Galactic plane is the brightest feature in both wavelength bands. While some of the emission is likely due to unresolved point 


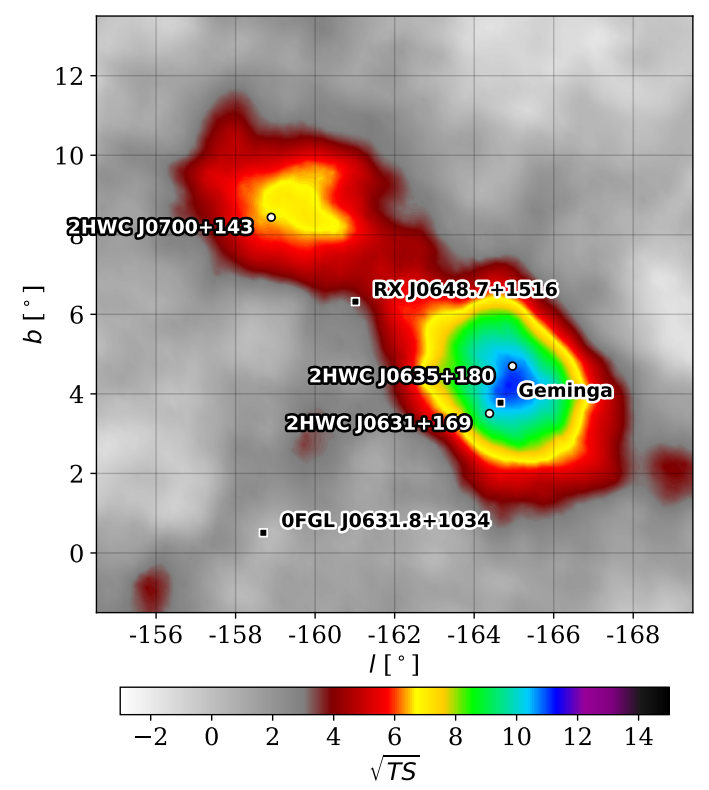

Figure 3: Map of significance in the region around Geminga (in Galactic coordinates). The TS values are for an extended source hypothesis represented by a disk of radius of $2.0^{\circ}$ with a spectral index of -2.0 . From Fig. 6 in Ref. [2].

sources, a large fraction of the gamma rays likely originate when the cosmic rays interact with gas and dust in the Galaxy. Gamma-ray observations are the most direct probe of the flux and spectrum of cosmic rays outside our solar neighborhood. Hadronic cosmic rays interacting with matter produce neutral pions that decay into gamma rays, whereas electrons create high-energy gamma rays through inverse Compton scattering with infrared photons and the cosmic microwave background. In addition, processes not directly related to cosmic-ray production may also contribute to the diffuse emission. For example, self-annihilating super-symmetric dark matter could play a significant role as an additional emission component with a distinct spectral signature.
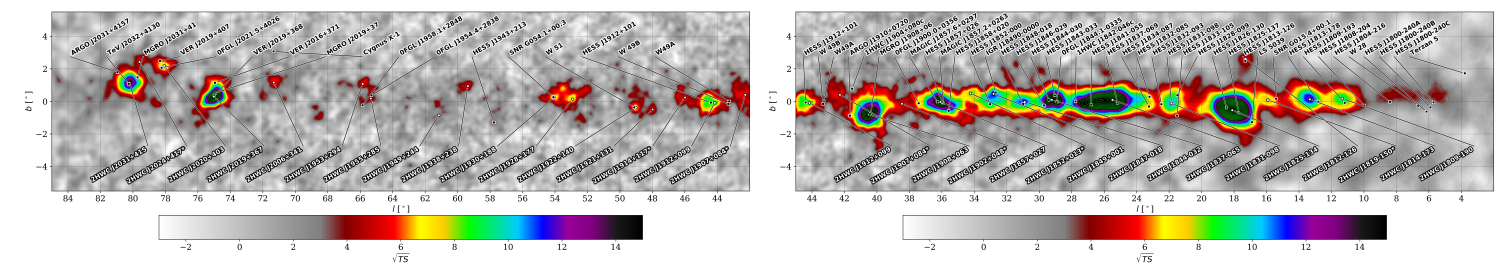

Figure 4: Map of significance of the Galactic Plane region in the FoV of HAWC in Galactic coordinates. The TS map corresponds to a point-source hypothesis with a spectral index of -2.7 . The green contour lines indicate values of $\sqrt{\mathrm{TS}}$ of $15,16,17$, etc. The $2 \mathrm{HWC}$ sources are represented by white circles and labels below the circle; whereas the source listed in TeVCat are represented with black squares and labels above the square symbol. From Figs. 8 and 9 in Ref. [2].

The fraction of the Galactic plane seen with HAWC is shown in Fig. 4. By observing nearly three orders of magnitude in energy, a multi-TeV survey instrument can map the diffuse emission in 
the Galaxy at multiple energies, and can provide the measurements to distinguish both the energy and spatial differences between the leptonic and hadronic emission mechanisms. The HAWC site is close to the equator and the field of view covers the inner Galaxy. HAWC observations provide data to study nearby regions such as Cygnus (at $1-2 \mathrm{kpc}$ ), as well as the more distant inner Galaxy (at about $10 \mathrm{kpc}$ ). The Cygnus region could be dominated by a very few cosmic-ray accelerators, whereas the cosmic rays from the inner Galaxy could come from a large collection of sources. These regions are hundreds of square degrees and require an instrument with a large field of view. A survey instrument in the southern hemisphere will allow us to extend these studies to the region toward the Galactic center.

Both the diffuse $\mathrm{GeV}$ and $\mathrm{TeV}$ gamma-ray fluxes are above predictions based on the assumption that local cosmic rays are representative of those elsewhere in the Galaxy. In order to match the EGRET data, the cosmic-ray density in the rest of the Galaxy must be two times higher than measured locally. Increasing the cosmic-ray density enough to match the Milagro data would violate the measured limits on the anti-proton flux. However, unresolved $\mathrm{TeV}$ sources may be contributing to the Milagro measurement of the flux from the Galactic plane.

For example, in the Cygnus region Milagro detects an excess, MGRO J2031+41, coincident with the largest matter density in the area. This Milagro source is also coincident with $\mathrm{TeV}$ J2032+41, observed by HEGRA, but the Milagro source is both brighter by a factor of 3 and more extended than the HEGRA source. Deep observations of the spatial and spectral morphology of this region with HAWC and IACTs will determine whether other sources exist in this region and whether the more localized $\mathrm{TeV}$ source could be the accelerator of protons which illuminate the entire region. In this way, the combination of the diffuse sensitivity of the proposed survey instrument with the deeper, higher angular resolution CTA follow-up observations will provide the most efficient way to map the entire Galactic plane over all angular scales.

\section{Monitoring the Transient Sky}

While AGNs (but not GRBs) have been observed in the TeV band, many questions surround the nature of $\mathrm{TeV}$ emission. For example, how does the $\mathrm{TeV}$ emission differ during the AGN quiescent state compared to the flaring state? What fraction of AGNs and GRBs emit GeV or TeV gamma rays? How much energy is available in these sources to accelerate cosmic rays? Continuous observations are needed to answer these questions because these sources are transient. This is possible using detectors with a large field of view, such as HAWC and the Fermi-LAT. While the Fermi-LAT is optimized to observe $\mathrm{GeV}$ gamma rays, its sensitivity above a few hundred $\mathrm{GeV}$ is limited by the physical size of the instrument $\left(0.8 \mathrm{~m}^{2}\right)$. A survey instrument can provide a large effective area at multi- $\mathrm{TeV}$ energies. These two types of instruments are therefore complementary in energy range, monitoring both the $\mathrm{GeV}$ and $\mathrm{TeV}$ skies.

\section{Multi-messenger searches}

Multi-wavelength and multi-messenger observations are essential to understanding not only the gamma-ray sky, but also many other astrophysical phenomena. A survey instrument can search 
the TeV sky in real time for flaring sources and provides rapid notifications of flares. This earlywarning system must be designed to enable observations at other wavelengths and with more sensitive $\mathrm{TeV}$ instruments. Currently HAWC provides a $\mathrm{TeV}$ flux or upper limit for steady sources in a search region of $8 \mathrm{sr}$, or about two-thirds of the sky. In addition to quickly reporting flares, HAWC also provides a synoptic survey of the TeV sky and produces sky maps of the observed TeV gamma-ray sources, as the one shown in Fig. 4. The proposed array will cover the southern sky with even higher sensitivity than HAWC.

The Fermi-LAT measures thousands of GeV gamma-ray sources, and many of these do not have obvious $\mathrm{TeV}$ counterparts. The proposed survey instrument will provide a natural extension of the energy reach of Fermi-LAT to the TeV scale for the sources in the southern sky. A widefield survey instrument will also be able to discover new $\mathrm{TeV}$ sources and monitor known sources. Follow-up observations with CTA can do a deep search for source variability on very short time scales, map the morphology of the sources, and constrain the spectra at lower energies than HAWC. The proposed detector can extend to energies above $10 \mathrm{TeV}$ the spectra measured by IACTs.

The importance of multi-messenger studies is demonstrated by the current efforts. For example, HAWC shares data with (and follows up on triggers from) many different experiments particle astrophysics including VERITAS, MAGIC, HESS, FACT, IceCube, ANTARES, SWIFT, FermiLAT, and LIGO. HAWC and IceCube, for example, observe the same range of energies and the same part of the sky in the northern hemisphere. The sources detected by HAWC provide excellent search candidates for neutrino emission by IceCube because proton cascades produce comparable fluxes to photons and neutrinos of similar energies. By providing target sources, the HAWC catalog improves the sensitivity of $\mathrm{TeV}$ neutrino observatories such as IceCube and ANTARES (as well as future observatories such as KM3Net) by more than a factor of two.

HAWC data are also being used to search for electromagnetic counterparts to the gravitational waves (GWs) observed by LIGO/VIRGO. The large field of view and high duty cycle provided by an array makes this type of detector well-suited to search for TeV counterparts to GW signals. This is particularly true because the current generation of $\mathrm{GW}$ observatories provide poor localization of GW events. Even when the future GW detectors go online, the localization of events in the southern hemisphere will continue to cover several square degrees.

\section{Probing Physics Beyond the Standard Model}

\subsection{Dark matter}

After decades of evidence in favor of the existence of Dark Matter (DM), its particle nature is still unknown. Two leading candidates for DM are Weakly Interacting Massive Particles (WIMPs), and Axion-like particles (ALPs). HAWC is being used to search for both WIMPs and axionlike particles. WIMPs are expected to create the gravitational wells of the Milky Way and its satellite galaxies, where they can interact and self-annihilate. If the WIMP mass is above $1 \mathrm{TeV}$, these interactions can produce $\mathrm{TeV}$ gamma rays. HAWC is sensitive to several high-mass WIMP models. Therefore, HAWC will either discover gamma-ray evidence for specific WIMP models or place strong limits on the dark matter mass and cross section above $1 \mathrm{TeV}$. The proposed detector will be several times more sensitive than HAWC, and it will be able to either discover evidence of DM, or improve on current limits. 
ALPs couple to gamma rays in the presence of magnetic fields. Therefore, gamma-ray conversions into ALPs would produce detectable signatures in the otherwise smooth spectra of gammaray sources like distant AGN. Axions may also be produced with neutron stars and then decay to gamma rays causing the neutron stars to appear as gamma-ray point sources. Gamma rays from high-redshift objects are expected to be attenuated by interactions with extragalactic background light. However, if $\mathrm{TeV}$ gamma rays oscillate into axions in the strong magnetic fields inside an AGN, they can pass unattenuated before oscillating back into $\mathrm{TeV}$ photons in the magnetic field of the Milky Way. Therefore, the observation of TeV gamma rays from very distant $(z \sim 1)$ AGNs can provide indirect evidence for the existence of ALPs.

\subsection{Lorentz invariance violation}

The combination of cosmological distances and rapid variability make short-duration transients such as gamma-ray bursts a unique laboratory to study the dependence of the speed of light on the energy of the photons. Theories of quantum gravity can predict the time delay for photons of a given energy traveling a known distance. At the maximum energy allowed by photon absorption on the extragalactic background light, a HAWC-like detector should be able to probe the scale of the Planck mass $\left(10^{19} \mathrm{GeV}\right)$ given a time delay of one second at $\mathrm{TeV}$ relative to simultaneous observations in the keV/MeV band. Observations of flaring of Mrk $501(z=0.034)$ on time scales of one minute with the MAGIC telescopes show evidence of such time delays which could be due to Lorentz invariance. However, a single measurement can only set a stringent limit. Multiple flares or bursts observed from sources at various redshifts would allow differentiation of source effects from a violation of Lorentz invariance.

\section{Serendipitous discoveries}

When wavelength bands are explored with unprecedented sensitivity, previously unknown sources and unknown types of sources are discovered. For example, the EGRET catalog contains over 150 previously unidentified sources, HESS has discovered several sources with no known counterparts, the Fermi-LAT saw two huge lobes of gamma-ray emission, Milagro has detected at least 3 new Galactic sources with no obvious counterpart, and HAWC has recently released its first catalog with previously unobserved $\mathrm{TeV}$ candidate sources. The discovery of new classes of objects unobserved at other wavelengths is a major strength of all-sky monitors. These serendipitous discoveries, while not possible to predict a priori, are frequently the most important scientifically. Examples of investigations with discovery potential with the proposed survey instrument are described in this poster.

\section{References}

[1] A.U. Abeysekara et al. [HAWC collaboration], Observation of the Crab Nebula with the HAWC Gamma-Ray Observatory, (2017) ApJ, 843, 39, [arXiv: 1701.01778]

[2] A.U. Abeysekara et al. [HAWC collaboration], The 2HWC HAWC Observatory Gamma-Ray Catalog, (2017) ApJ, 843, 40, [arXiv: 1701 .02992] 